\title{
A Quantum Neural Network Approach for Portfolio Selection
}

\author{
R.P Mahajan \\ School of Computer Science, Devi Ahilya Vishwa Vidyalaya, Indore-452001, India \\ Faculty at Dept. of Computer Science \& Engineering, Institute of Engineering \& Science, \\ IPS Academy, Indore-452012, India
}

\begin{abstract}
A new field of computation is emerging which integrates quantum and classical computation. This is applied to solve the financial engineering problem of portfolio selection. Hopfield neural network is used for portfolio selection. A quantum inspired hybrid model of quantum neurons and classical neurons is proposed for the prediction of stock prices. An effort is made, probably the first time to develop and use a hybrid quantum neural network for the prediction of stock prices. The suggested multilayer hybrid quantum neural network contains hidden layer of quantum neurons while the visible layer is of classical neurons. The asset distribution is done by a modified greedy algorithm. It is assumed that quantum computers when come into existence shall provide huge potential in the form of computational power and memory. Classical Neural networks $(\mathrm{CNN})$ have shown tremendous acceptability in solving problems with non-linear formulations that requires huge processing power and large memory which a quantum computer can provide, when they will come into existence.
\end{abstract}

\section{Keywords}

Quantum neural network, portfolio selection, resource allocation, stock price prediction, investment weights, quantum back propagation, quantum computation.

\section{INTRODUCTION}

The allocation of asset, in an investment decision, is a task on which a lot of work is being done. The Markowitz[1] mean variance approach has found a wide spread acceptability in various forms. The expected return of a portfolio is described using the mean returns of the assets and the portfolio risk is represented by the covariance matrix of the asset returns. The objective of the investor is to select a portfolio which maximizes his returns with minimum risk involved. The mean variance model is required to satisfy two conflicting optimization criterion, one which minimizes risk and the other with predetermined minimum return. Moreover, it is desirable that the selected portfolio shall have a restricted number of assets, invest a minimum portion of available resources in each selected asset, and invest a maximum portion in a single asset. With such restriction the efficient frontier search becomes difficult and complex. Chang et al.[2] further extended the mean variance model by including cardinality constraints that limit a portfolio to have a specified number of assets, and to impose limits on the proportion of the portfolio held in a given asset.

Alberto Fernandez[3] have used Hopfield Neural Network for finding the efficient frontier. He further used heuristic method to allocate fund to various assets of the portfolio.

Benioff and Feynman[4,5] has proposed the concept of quantum computation. Quantum algorithms by P. W. Shor and L.K. Grover[6,7] has received a lot of attraction. The idea of quantum neural network(QNN) can take leverage from quantum computation in the field of artificial intelligence, Many prototypes for QNN similar to classical neural networks have been proposed. Kak[8] was first to present concept of QNN. Gupta and Gia[9] has shown that QNN has almost the same computational power as CNN. Menneer and Narayan[10,11] has laid some foundation of basic concepts inspired by quantum theory for use in neural networks design, development and implementation. It has been further argued that, a fully QNN has no advantage over a hybrid QNN and may produce worst results. Quantum hidden units are plausible as they are not in direct contact with the real world[10,11].

Ezonov and Ventura[12] have introduced the possibilities of combining the unique computational capabilities of $\mathrm{CNN}$ and quantum computation. This combination can produce a computational paradigm of incredible potential. Xiao and Cao[13] have proposed a QNN model based on quantum and classical neurons. Miszczak[14] has proposed ways for preparing initial quantum state based on probabilities and Nielsen et. al [15] has given concepts of quantum computation.

Many Worlds approach proposed by Everett [16] to quantum mechanics has caught the attention of physicist. Based on many worlds, ensemble computers are being researched. Long et al [17] has proposed parallel quantum computing mode for ensemble quantum computer. Further, Vizzotto et al [18] has proposed that the parallel ensemble quantum computer possesses, besides quantum parallelism, a kind of classical single-instruction-multiple-data parallelism. It explores additional speedup by making quantum computers working in parallel.

Thus, quantum computers may be produced with inbuilt parallelism and large memories. Visualizing these potential for quantum computers, it is considered that the QNN shall be the next natural step in the evolution of neuro-computing system.

Since last two decades, a great attention is visualized in the efforts of researcher on the idea of time series prediction. They have studied these fluctuations through the use of CNN. Various prediction strategies can be applied for improving accuracy of prediction. A classical feed forward back propagation neural network has been successively applied and tested in this paper to the time series of one previous prediction using multiple stocks data using sliding window [19].

This paper has proposed and applied, a hybrid three layer feed forward back propagation quantum inspired neural network for stock price prediction. The input layer is classical, the hidden layer is quantum neuron, and the output layer is classical. The output calculation is based on classical computation. Input and output to the hybrid QNN are classical as they have to interact with the real world.

The predicted output from classical and quantum neural networks are compared and discussed with respect to actual. 
The suggested model for the portfolio selection problem generates efficient frontier using classical Hopfield neural network based on modified Markowitz model. The predicted stock values using the quantum neural network are generated. These are used to distribute the fund in various assets of the portfolio using a modified heuristic approach. The return and risk are compared for greedy and modified greedy heuristic approach.

The section 2 deals with the hybrid quantum inspired neural network, section 3 deals with learning in quantum neural network, section 4deals with algorithm for hybrid quantum inspired back propagation, section 5 describes the Markowitz model, section 6 deals with Hopfield neural network, section 7 discusses the Hopfield portfolio selection problem, section 8 deals with stock price prediction, section 9 deals with fund distribution among the assets, while section 10 presents results and discussion followed by conclusion in section 11 .

\section{HYBRID QUANTUM INSPIRED NEURAL} NETWORK

The QNN is based on the techniques of quantum computation. Qubit is defined as the smallest unit of information in quantum computation which is a probabilistic representation. A qubit may either be in the " 1 " or " 0 " or in any superposition of the two [9].

Figure 1 and 2 represent the concept of hybrid quantum neural network.

The state of the qubit can be represented as:

$|\psi\rangle=\alpha|0\rangle+\beta|1\rangle$

Where $\alpha$ and $\beta$ are the numbers that indicate the amplitude of the corresponding states such that:

$|\alpha|^{2}+|\beta|^{2}=1$.

A qubit is defined as smallest unit of information in quantum computation. It is defined as a pair of numbers $\left[\begin{array}{l}\alpha \\ \beta\end{array}\right]$.

Angle $\theta$, a more geometrical aspects is defined such that

$$
\cos (\theta)=|\alpha| \text { and } \sin (\theta)=|\beta| ;
$$

Quantum gates may be applied for modifying the probabilities as a result of weight updating,. One such rotation gate can be :

$U(\Delta \theta)=\left[\begin{array}{cc}\cos (\Delta \theta) & -\sin (\Delta \theta) \\ \sin (\Delta \theta) & \cos (\Delta \theta)\end{array}\right]$

The following hybrid quantum inspired neural network is proposed for the stock's price prediction.

\section{- Initialize}

\section{a. Quantum hidden neuron:}

Start from state $|0\rangle$, prepare the superposition :

$$
\sqrt{p}|0\rangle+\sqrt{1-p}|1\rangle \quad \text { with } 0 \leq|\mathrm{p}| \leq 1 \text {; }
$$

Where $\mathrm{p}$ represents random probability of initializing the system in the state $|0\rangle$.

The desired state can be reached by using rotation gate $\mathrm{R}$ :

$$
\begin{aligned}
& \mathbf{R}(\theta)=\left[\begin{array}{rr}
\cos (\theta) & -\sin (\theta) \\
\sin (\theta) & \cos (\theta)
\end{array}\right] ; \\
& \tan (\theta)=\sqrt{\frac{p}{1-p}} ; \\
& \theta=\arctan \sqrt{\frac{p}{1-p}} ;
\end{aligned}
$$

$$
\begin{aligned}
\mathrm{R}(\theta) & =\left[\begin{array}{cc}
\sqrt{1-p} & -\sqrt{p} \\
\sqrt{p} & \sqrt{1-p}
\end{array}\right] ; \\
{\left[\begin{array}{l}
\alpha \\
\beta
\end{array}\right] } & =\left[\begin{array}{cc}
\sqrt{1-p} & -\sqrt{p} \\
\sqrt{p} & \sqrt{1-p}
\end{array}\right]\left[\begin{array}{l}
1 \\
0
\end{array}\right]
\end{aligned}
$$

b. Classical neurons:

Initialize classical neurons by random number generation.

- Output from quantum neuron

$$
v_{j=f\left(\sum_{i=1}^{n} w_{j i} x_{i}\right)} \text {. }
$$

Where $\mathrm{f}$ is a problem dependent sigmoid or Gaussian function

- Output from the network:

$$
y_{k}=f\left(\sum_{j=1}^{l} w_{j k} *\right.
$$

vj).

- The desired output is $o_{k}$ the corresponding squared error is:

$$
E_{k}^{2}=\frac{1}{2}\left|y_{k}-o_{k}\right|^{2}
$$

\section{LEARNING IN QUANTUM NEURAL NETWORK}

The learning follows the rules of feed forward back propagation algorithm

- Updation of output layer weight $\Delta w_{j k}=\eta e_{k} f^{\prime} v_{j} \ldots \ldots \ldots \ldots \ldots \ldots \ldots$. (vii)

- Updation of quantum hidden layer weight

in quantum BP algorithm the weights are updated by quantum gate according to equation (iii), in this case the equation shall be

$\left[\begin{array}{c}\alpha_{\mathrm{ij}}{ }^{\prime} \\ \beta_{\mathrm{ij}}{ }^{\prime}\end{array}\right]=\left[\begin{array}{cc}\cos \left(\Delta \theta_{\mathrm{ij}}\right) & -\sin \left(\Delta \theta_{\mathrm{ij}}\right) \\ \sin \left(\Delta \theta_{\mathrm{ij}}\right) & \cos \left(\Delta \theta_{\mathrm{ij}}\right)\end{array}\right]\left[\begin{array}{c}\alpha_{\mathrm{ij}} \\ \beta_{\mathrm{ij}}\end{array}\right] . .(\mathrm{viii})$ where $\Delta \theta_{\mathrm{ij}}=-\frac{\partial \mathrm{E}}{\partial \theta_{\mathrm{ij}}}$;

$=-\frac{\partial \mathrm{E}}{\partial_{y_{k}}} \frac{\partial_{y_{k}}}{\partial_{v_{j}}} \frac{\partial_{v_{j}}}{\partial_{\theta_{\mathrm{ij}}}}$ by chain rule

From equations vi, $v$ and iv:

$=-E_{k} f^{\prime} w_{j k} v_{j} x_{i}\left(\cos \left(\gamma_{i j}\right)-\sin \left(\gamma_{i j}\right)\right)$

where $\gamma_{i j}$ is a phase of $\left|\psi_{i j}\right\rangle$ such that

$$
\left|\psi_{i j}\right\rangle=\left[\begin{array}{l}
\cos \left(\gamma_{i j}\right) \\
\sin \left(\gamma_{i j}\right)
\end{array}\right]
$$

for $\gamma_{i j}$ updation shall be:

$\gamma_{i j}{ }^{\prime}=\gamma_{i j+} \eta \Delta \theta_{\mathrm{ij}} ; \quad \eta$ is learning rate 


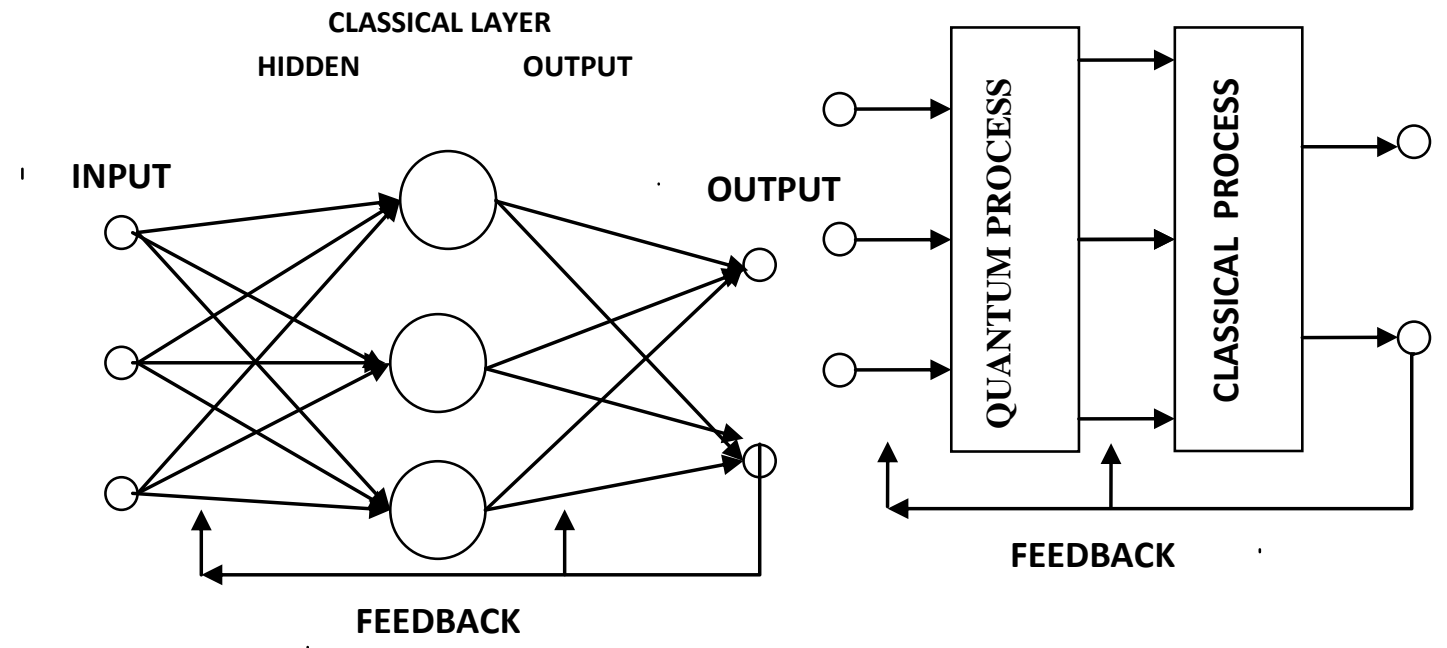

Figure 1. (A) Classical and (B) Hybrid Quantum Neural network Learning by Feed Back

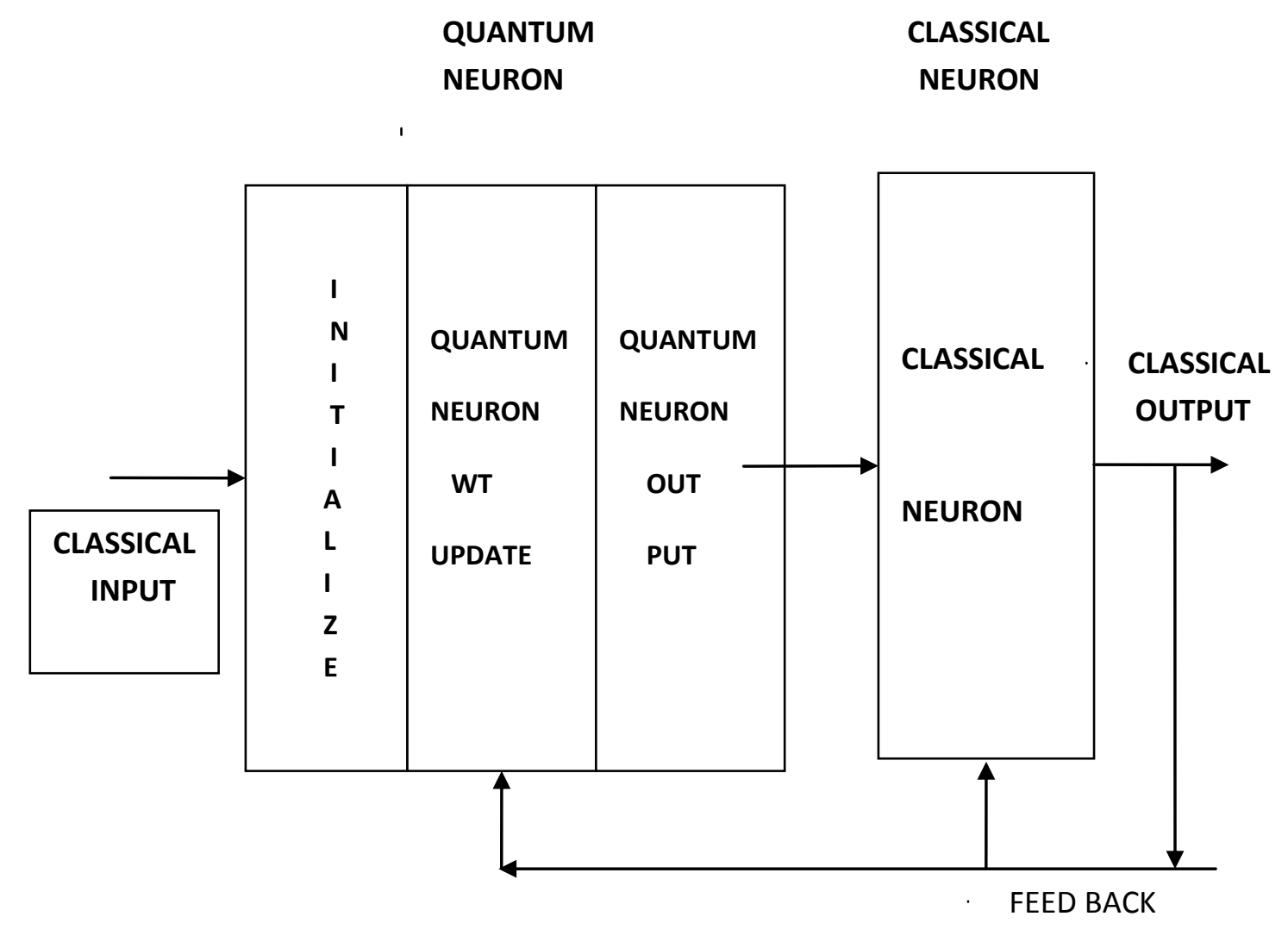

Figure 2. Hybrid Quantum Neural Network

\section{ALGORITHM: QUANTUM INSPIRED HYBRID BACK PROPAGATION}

Start with randomly chosen weights for classical output neurons and initialize quantum hidden neuron

While mean squared error is

computational bounds not exceeded 
Do

For each input array $\mathrm{x}_{1}, \ldots \ldots \ldots, \mathrm{x}_{\mathrm{n}}$

Compute hybrid quantum hidden node output

Compute the network output

Compute the output error

Modify weights between hidden and output node

Apply qui Im gate and modify hidden node weights

\section{End For}

End Do

\section{End while}

End

\section{THE BASIC MODEL: MARKOWITZ MODEL}

For the portfolio selection problem in canonical form it is desired to find a portfolio that minimizes the risk at given levels of return rate. In the Markowitz formulation the risk measure is given by the variance of the portfolio. This measure is the objective function most commonly used in related works. The Markowitz model (1952) is as follows:

Minimise $\lambda\left[\sum_{\mathrm{i}=1}^{\mathrm{N}} \sum_{\mathrm{j}=1}^{\mathrm{N}} \mathrm{x}_{\mathrm{i}} \sigma_{\mathrm{ij}} \mathrm{x}_{\mathrm{j}}+(1-\lambda)\left[\sum_{\mathrm{i}=1}^{\mathrm{N}}-\mu_{\mathrm{i}} \mathrm{x}_{\mathrm{i}}\right]\right]$

Subject to:

$\sum_{\mathrm{i}=1}^{\mathrm{N}} \mathrm{x}_{\mathrm{i}}=1 \quad$ where $0 \leq \mathrm{x}_{\mathrm{i}} \leq 1 ; \mathrm{i}=1$ to $\mathrm{N}$

Where

$\mathrm{N}$ = number of different assets

$\mu_{\mathrm{i}}=$ mean return of asset $\mathrm{i}$

$\sigma_{\mathrm{ij}}=$ co - variance between return of asset i $\& j$

$\lambda=$ risk aversion papameter and $\lambda \in(0,1)$

$\lambda=0$ represent maximising the portfolio mean return

$\lambda=1$ represent minimising the variance

$\mathrm{x}_{\mathrm{i}}=$ proportion of capital in asset $\mathrm{i}$

To generalize the standard Markowitz model to include cardinality and bounding constraints, a models formulation [2] follows:

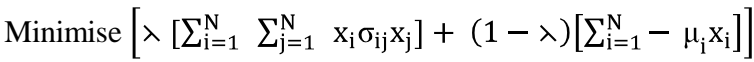

Subject to:

- $\quad \sum_{\mathrm{i}=1}^{\mathrm{N}} \mathrm{x}_{\mathrm{i}}=1$

- $0 \leq \mathrm{x}_{\mathrm{i}} \leq 1 ; \mathrm{i}=1$ to $\mathrm{N}$

- $\quad \sum_{\mathrm{i}=1}^{\mathrm{N}} \mathrm{z}_{\mathrm{i}}=\mathrm{k} ;$ where $\mathrm{z}_{\mathrm{i}} \in(0,1)$

- $\mathrm{z}_{\mathrm{i}}$ is additional decision variable 1 if asset $i$ to be included 0 if asset $i$ to

be excluded

- $\mathrm{k}=$ desired number of different asset

- $\quad \varepsilon_{\mathrm{i}}=$ lower bounds for the proportion of capital to be invested in asset $\mathrm{i}$

- $\partial_{\mathrm{i}}=$ upper bounds for the proportion of capital to be invested in asset i

\section{HOPFILED NEURAL NETWORK:}

Hopfield net is a form of recurrent artificial neural network and it serves as auto-associator and optimizer. The units in Hopfield nets are binary threshold i.e the units take on two different values for states and the value is determined by whether or not the units input exeed their threshold. Hopfield net can either have units that take on values 0 or 1 .

In Hopfield neural network the state is made up of $\mathrm{N}$ neurons, the state of the network is defined by the vector:

$\mathrm{X}=\left[\mathrm{x}_{1}, \mathrm{x}_{2}, \mathrm{x}_{3}, \mathrm{x}_{4}, \ldots \ldots, \mathrm{x}_{\mathrm{N}}\right]^{\mathrm{T}}$

$\mathrm{x}_{\mathrm{i}}=1$ or 0

The alteration in the state of Hopfield model causes monotonic decrement in Hamiltonian energy or Lyapunov function.

$E=-\frac{1}{2} \sum_{i, j} w_{i, j} x_{i} x_{j}+\sum_{j} \theta_{j} x_{j}$ ..(i)

$\mathrm{w}_{\mathrm{i}, \mathrm{j}}$ is the strength of the connection weight from unit $\mathrm{j}$ to unit i(the weight of the connection)

$x_{i}$ is the state of unit $i$
$\theta_{i}$ is the threshold of unit $i$

The induced local field $\mathrm{v}_{\mathrm{j}}$ is defined by:

$v_{j}=\sum_{i=1}^{N} w_{j, i} x_{i}+\theta_{j}$

Neuron $\mathrm{j}$ is modified according to the deterministic rule:

$x_{j} \begin{cases}1 & \text { if } v_{j}>0 \\ 0 & \text { if } v_{j} \leq 0\end{cases}$

Or $\mathrm{x}_{\mathrm{j}}=\operatorname{Sigmoid}\left[v_{j}\right]$

$\mathrm{w}_{\mathrm{i}, \mathrm{i}}=0$, for all $\mathrm{i}$ (no unit has connection to itself)

$\mathrm{w}_{\mathrm{i}, \mathrm{j}}=\mathrm{w}_{\mathrm{j}, \mathrm{i}} \quad$ (connection are symmetric )

Thus Hopfield neural network are a class of densely connected non-linear network of perceptrons. Because of its non-linearity, a number of undesirable local minima also occur. If the neuron update rule is probabilistic and of a particular form, the problem of local minima can be diminished.

For solving optimization problem, the Hopfield neural networks belong to the penalty method due to their recurrent single layer architecture without any hidden units.

Now, if the equilibrium points were preselected (for instance by hard coding the weights), then the system can work as an autoassociator and optimizer.

Hopfieled neural network is hardware implementable. It is massively parallel, special purpose and can be used to solve linear programming problems, quadratic programming problem and other hard problem.

\section{HOPFIELD PORTFOLIO SELECTION PROBLEM}

The objective function for the selection of the portfolio is:

$f(x)=\left[\lambda\left[\sum_{i=1}^{N} \sum_{j=1}^{N} x_{i} \sigma_{i j} x_{j}\right]+(1-\lambda)\left[\sum_{i=1}^{N}-\mu_{i} x_{i}\right]\right]$

Passing the multiplicative coefficients inside the additions:

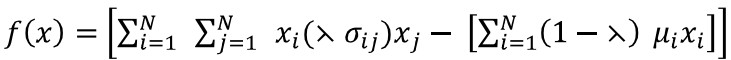

Multiply and divide by -2

$f(x)=\quad-\frac{1}{2}\left[\sum_{i=1}^{N} \sum_{j=1}^{N} x_{i}\left(-2 \lambda \sigma_{i j}\right) x_{j}-\left[\sum_{i=1}^{N}(1-\right.\right.$
$\left.\left.\lambda) \mu_{i} x_{i}\right]\right]$

Comparing this with energy function of the Hopfield model, it can be translated into : 


$$
\begin{array}{lrr}
E(x)= & -\frac{1}{2}\left[\sum_{i=1}^{N} \sum_{j=1}^{N} x_{i}\left(\lambda \sigma_{i j}\right) x_{j}-\quad\left[\sum_{i=1}^{N}(1-\right.\right. \\
\lambda & =-\frac{1}{2}\left[\sum_{i=1}^{N} \sum_{j=1}^{N} x_{i} w_{i j} x_{j}-\left[\sum_{i=1}^{N} b_{i} x_{i}\right]\right]
\end{array}
$$

Where:

$\mathrm{w}_{\mathrm{ij}}=-2 \lambda \sigma_{\mathrm{ij}}$

$b_{i}=(1-\lambda) \mu_{i}$

The constraint $\mathrm{x}_{\mathrm{i}} \in\left(\varepsilon_{i}, \partial_{i}\right)$ ( $\mathrm{x}_{\mathrm{i}}$ belogs to lower and upper limit) shall be satisfied using a sigmoid threshold function since its output already lie inside the desired interval.

The cardinality constraint $\sum_{i=\mathbf{1}}^{N} \boldsymbol{z}_{i}=\boldsymbol{k}$; where $\boldsymbol{z}_{\boldsymbol{i}} \in$ $(\mathbf{0}, \mathbf{1})$, the neural network has been used that has $\mathrm{N}$ neurons that will follow Hopfield dynamics and the selected $\mathrm{K}$ neurons shall be having 1 as output and rest shall be having 0 outputs. Shall the user require still less stocks in his portfolio, the same can be obtained by pruning the network.

\section{PORTFOLIO STOCK PRICE PREDICTION}

The efficient frontier generated from Hopfield neural network shall effectively contribute for desired return. The individual stocks selected are the elements that constitute the target portfolio.

The succession of values in a time series is usually influenced by a number of external information. When this information is not available only past value of the series itself can be used to build a prediction model.

$\mathrm{X}_{\mathrm{t}+1}=\mathrm{f}\left(\mathrm{x}_{1}, \ldots \ldots \ldots, \mathrm{x}_{\mathrm{n}}\right)$; where $\mathrm{x}_{\mathrm{t}+1}$ is estimated next value based on current and past values of $\mathrm{x}$.

Efficient market hypothesis (EMH) emphasizes that if statistically significant serial dependencies exist within time series of stock prices, the community of business analyst will immediately exploit it. Stock price changes can therefore be only be explained by arrival of new information, which by definition cannot be forecasted. EMH is only true when linear models are applied. It has been generally established that by use of non-linear models like neural networks, the results challenge the EMH hypothesis.

The data used for stock price prediction are daily closing prices of 10 stocks. A total of 7 years (2003-2010) daily data were considered [20]. The figure 3(3.1 to 3.4) illustrates the price behavior of these stocks and illustrates the prediction performance of each stock while comparing classical neural network stock price prediction with that of quantum neural network.

\section{FUND DISTRIBUTION AMONG} ASSETS

The constraint $\sum_{i=1}^{N} x_{i}=1$; can be satisfied through a heuristic algorithm in [2]. The feasibility of the portfolio is evaluated using a greedy algorithm. The existing algorithm is modified to enhance the performance of the portfolio. The algorithm allocates the minimum percentage to each asset followed by allocation of maximum allowable ratio to the entire asset with high return. This ensures that all the constraint relating to lower and upper bounds are satisfied. In the next iterations the algorithm allocates free proportions from the assets having returns more than the allocable proportion to all other assets. The allocation to a particular asset depends on the return of that asset. In a case where fund remains un-allotted after allocation following the lower and upper bound constraints rule, either the upper bounds are revised or the asset with higher return may be used for further allotment.

\section{RESULTS AND DISCUSSIONS}

The Hopfield neural network model is used to generate the efficient frontier. The efficient frontier so generated from the past data is input to the hybrid quantum back propagation model.

The hybrid quantum back propagation model uses 30 quantum hidden neurons and 30 classical output neurons. The performance of the hybrid quantum model has been compared with classical back propagation neural network model having the same number of hidden and output neurons, parameters and data.

The ability of neural networks to discover nonlinear relationships in input data makes them ideal for modeling nonlinear dynamic systems of the stock market has been established. It has been found that the average error using lots of data is smaller than that using less amount of data. That is, the more data for training the neural network, the better prediction it gives. If the training error is low, predicted predictive stock prices are close to the real values.

The back propagation network has performed and predicted stock price trends and thus further strengthens the challenges to the EMH. Since, if a neural network can outperform the market consistently or predict its direction with reasonable accuracy, the validity of the EMH is questionable.

The objective of this study was to establish the fact that quantum neural network can perform with almost the same accuracy as the classical ones. Even though, our hybrid quantum model uses quantum hidden neurons containing single qubit registers and interfaces with its classical component. All the results, in the figure 3 for four stocks show that there is a close matching of results in both the cases with slight improvements (around $4 \%$ ) using QNN.

It can be further concluded that the processing time in quantum neural networks shall be very small due to the inbuilt capacity of the quantum computers to process the data in parallel. It is evident from the structure of the neural network that the calculations in all the neurons of the hidden layer can be performed concurrently. In this hidden layer the forward and back propagation calculation shall be faster. With the concept of copies of quantum processors, the calculations for the inner layer output can be performed in a single iterations as compared to $\mathrm{n}^{2}$ iterations in case of $\mathrm{CNN}$. Quantum computers can have huge memories associated with them and thus the future quantum neural network shall be able to handle large neural networks, thus ensuring the coverage of a wide range of stocks across all corners of stock markets globally.

The modified heuristic greedy algorithm is presented for maximizing the return of the portfolio by efficient allocation of the fund. The algorithm has reduced the risk by $3.61 \%$ and improved the return by around $1.38 \%$. Fig. 4 presents the return and variance of the portfolio and compares the output of the modified algorithm. 


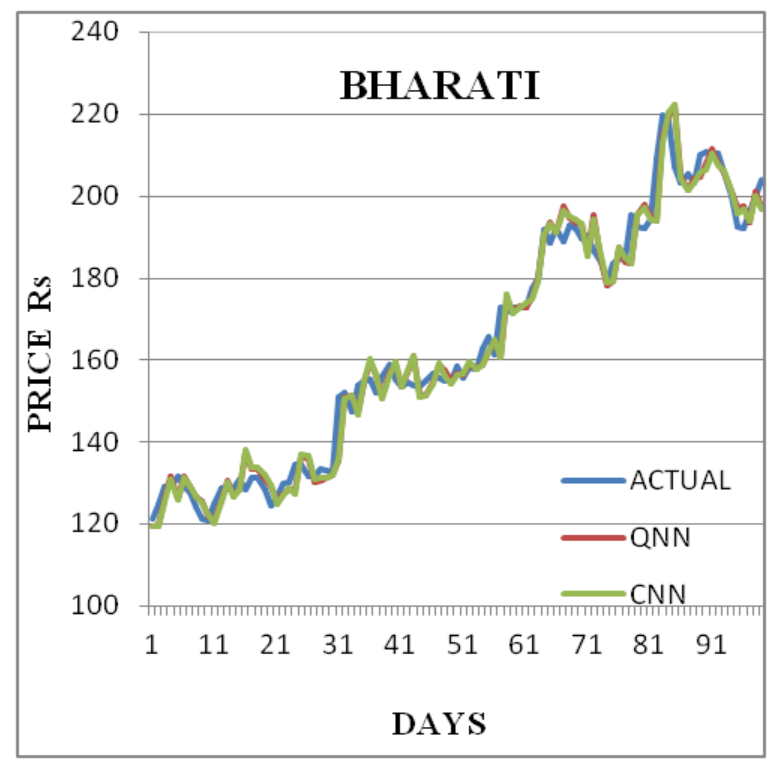

(A)

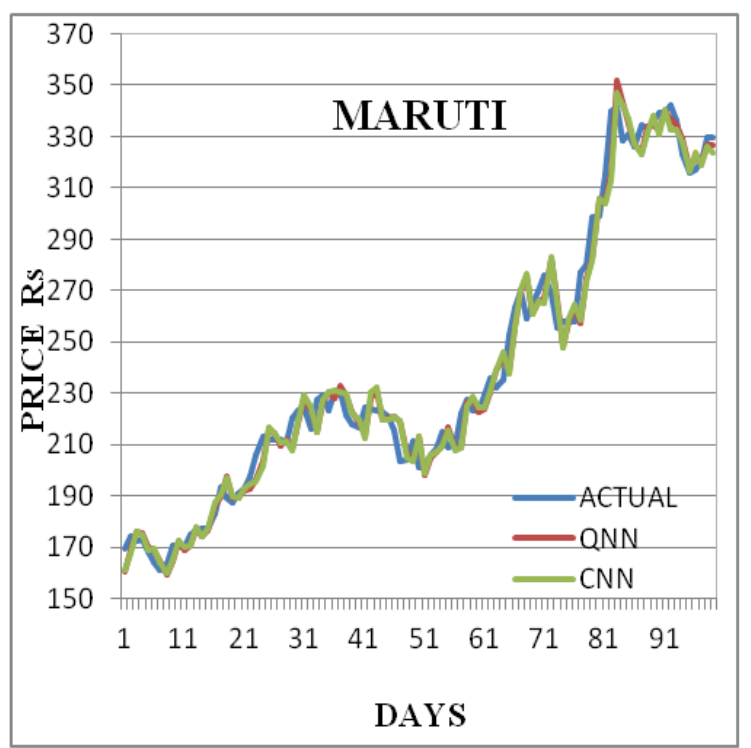

(C)

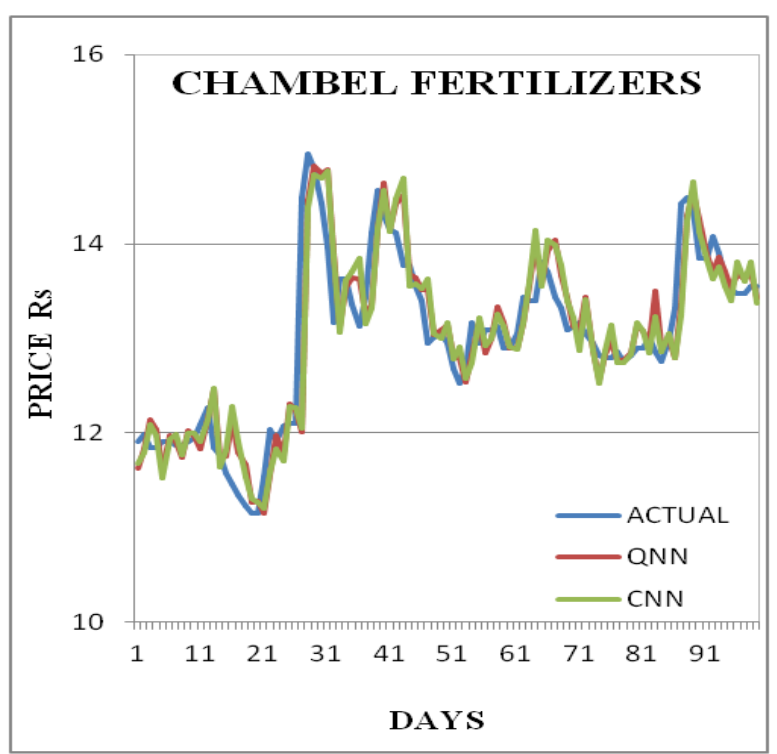

(B)

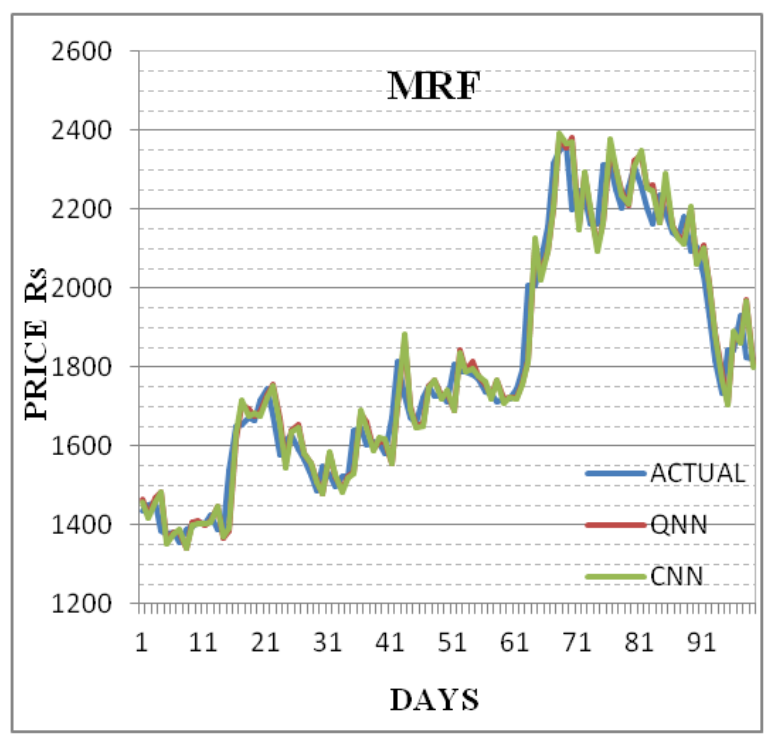

(D)

Figure 3. Comparative Prediction of stock prices using CNN and hybrid QNN with actual prices 


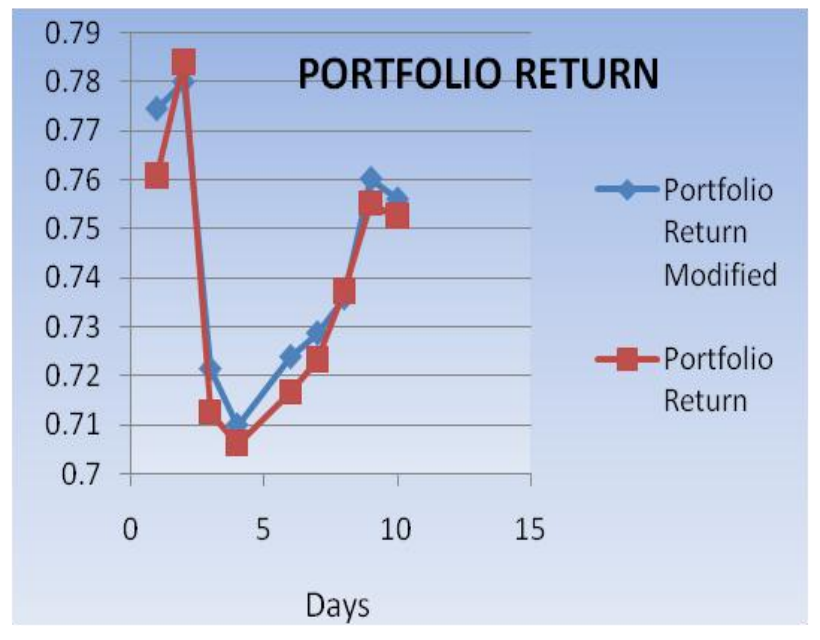

Fig. 4 Portfolio Variance and Return comparison

Finally it is observed that:

- $\quad$ The prices move in a

- $\quad$ close range with an improvement of about $4 \%$ using QNN over CNN.

- $\quad$ Processing time will reduce due to quantum parallelism [18].

- Availability of huge quantum memory will ensure coverage for a wide range of stocks.

- The heuristic greedy algorithm has increased the return by $1.38 \%$ and reduced the risk (variance) by $3.61 \%$ for the selected portfolio.

The comparison of results between classical and quantum neural networks has revealed that the results match closely and QNN results are better.

\section{CONCLUSION}

The portfolio slection problem has been dealt by generating the efficient portfolio using the Hopfiled neural network. The returns from the stock are predicted using the hybrid quantum neural network. The asset distribution has been done by using a modified heuristic algorithm resulting in reduced risk by $3.61 \%$ and increased return by $1.38 \%$.

The QNN result has established that classical algorithms can be successfully replaced by hybrid QNN when quantum computers become a reality with improved performance $4 \%$ prediction) and enhanced speed. It can be further concluded that the processing time in quantum neural networks shall be very small due to the inbuilt capacity of the quantum computers to process the data in parallel. It is evident from the structure of the neural network that the calculations in all the neurons of the layer can be performed concurrently. The advantage of quantum computing can be exploited by extending the quantum computation concepts to recurrent Hopfield nets and stochastic Boltzmann neural networks. The extension so achieved can be applied to the solution of combinatorial problems in financial engineering.

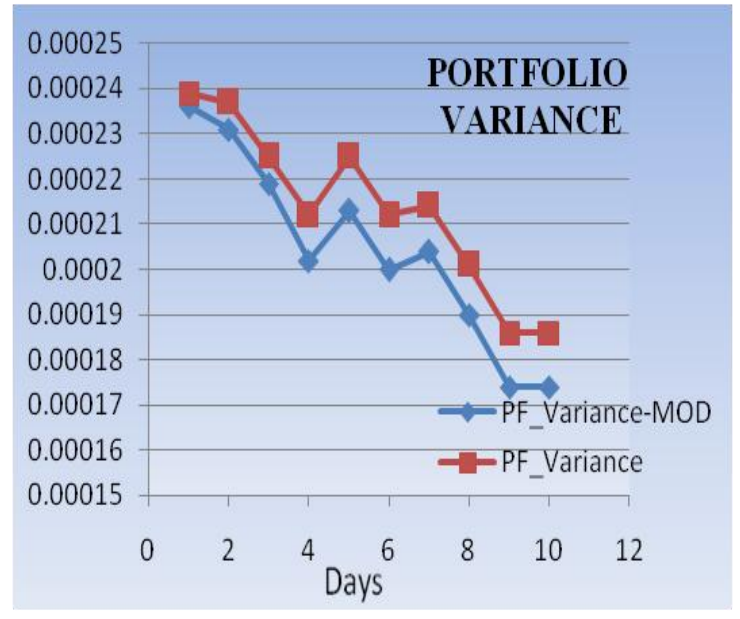

\section{ACKNOWLEDGEMENT}

The author is deeply grateful to his $\mathrm{PhD}$ guide Dr Raj Kamal for hid inspiration and guidance. He is also grateful to Dr ML Sharma for their continuous motivation, guidance and technical discussions.

\section{REFERENCES}

[1] H. Markowitz, "Portfolio Selection", The Journal of Finance, Vol. 7, No. 1. (Mar., 1952), pp. 77-91.

[2] TJ Chang et. al, "Heuristic for Heuristics for cardinality constrained portfolio optimization", Computer and Operations Research 27(2000) 1271-1302.

[3] Alberto Fernández, Sergio Gómez, "Portfolio selection using neural networks", http://arxiv.org/ftp/cs/papers/0501/0501005.pdf, 2005

[4] P. Benioff, "The computer as physical system: A microscopic Quantum Mechanical Hamiltonian model of computers as represented by turing machine," Journal of Statistical Physics, vol. 22, pp. 563-591, 1980.

[5] R. Feynman, "Simulating physics with computers", International Journal of Theoretical. Physics, 21, pp 467-488, 1982

[6] Shor, Peter W., "Polynomial-time algorithms for prime factorization and discrete logarithms on a quantum computer", SIAM Journal on Computing, Vol. 26, pp. 1484 - 1509, October 1997.

[7] Lov K. Grover., "A fast quantum mechanical algorithm for database search", In Proceedings of the 28th ACM STOC, pages 212-219, 1996.

[8] SC Kak, "Quantum neural computing", Advances in Imaging and Electron Physics 94, pp. 259-314, 1995.

[9] S. Gupta and R. Zia, "Quantum neural networks", Technical report, Available:http://www.arxiv.org/PS_cache /quant- ph/ pdf/ 0201/ 0201144.pdf, 2002.

[10] T. Menneer, "Quantum Artificial Neural Networks", $\mathrm{PhD}$ thesis, University of Exeter, 1998. 
[11] Ajit Narayanan and Tammy Menneer, "Quantum artificial neural network architectures and components.", Information Sciences, volume 124 nos. 1-4, pages 231255, 2000.

[12] Ezhov, A. and D. Ventura, "Quantum neural networks", in: N. Kasabov(ed) Future Directions for Intelligent Systems and Information Sciences, Springer Verlag 2000.

[13] Hong Xiao and M. Cao, "Hybrid quantum neural networks model algorithm and simulation" in the proceedings of the fifth International Conference on Neural Computation Tiaingiin, china, 2009.

[14] J. A. Miszczak. "Initialisation of quantum registers based on probability distribution." Technical report, Available: http://zksi.iitis.gliwice.pl/wiki/projects:kulka IITiS PAN, 2007.

[15] Michael A. Nielsen and Isaac L. Chuang, "Quantum computation and quantum information", Cambridge University Press, 2000.

[16] Everett H., "The theory of the universal wave function. In N. Graham \& B. DeWitt (Eds.)", The many-worlds interpretation of Quantum Mechanics (3-140). Princeton: Princeton University Press (1973).

[17] Gui Lu Long, Li Xiao, "Parallel Quantum Computing in a Single Ensemble Quantum Computer", Physical Review A 69, 052303 (2004)
[18] Juliana Kaizer Vizzotto and André Rauber Du Bois, Modeling Parallel Quantum Computing Using Transactional Memory, Electronic Notes in Theoretical Computer Science, Volume 270, Issue 1, 10 February 2011, Pages 183-190

[19] FDD Freitas et. al, "Portfolio Selection with Predicted Returns Using Neural Networks", ACM-DL , Expert System with Applications vol 35 issue 1-2, July 2008

[20] http://finance.yahoo.com/ BSE (Bombay Stock Exchange)

\section{AUTHORS PROFILE}

Prof. R.P. Mahajan received the B.E. degree from S.G.S.I.T.S. Indore, India in 1972, M.E. from Indian Institute of Science, Bangalore, India in 1974 and M.Tech. from BIT Mesra, Ranchi, India in 1999. Presently he is a Professor in the department of Computer Sc. \& Engg., Institute of Engg. \& Sc., IPS Academy, Indore and is a Ph.D candidate from School of Computer Science, Devi Ahilya Vishwa Vidyalya, Indore.

Since 1974 he worked for various positions in industry and academics. His research interest includes CAD, DBMS, distributed systems, AI and its applications in finance \& engineering. 